\title{
Experience in the Management of Patients with Chronic Vascular Ulcers of the Lower Limbs Using Negatively Charged Polystyrene Microspheres
}

\author{
María Teresa Cacua Sánchez \\ Peripheral Vascular Surgeon Universidad El Bosque, Specialist in Management and Health Services, Universidad Sergio Arboleda, \\ Bogotá, Colombia
}

Correspondence should be addressed to María Teresa Cacua Sánchez; mcacua@gmail.com

Received 17 July 2019; Accepted 18 October 2019; Published 22 January 2020

Academic Editor: Antonio Bozzani

Copyright ( 2020 María Teresa Cacua Sánchez. This is an open access article distributed under the Creative Commons Attribution License, which permits unrestricted use, distribution, and reproduction in any medium, provided the original work is properly cited.

Background. Chronic ulcers of the lower limbs are a socioeconomic health problem, having a high incidence in the adult population. Despite a correct etiological treatment, in addition to the multiple lesion management options available, healing percentage and speed remain low, which makes it a great therapeutic challenge. Objective. To describe the outcome and effectiveness of the use of PolyHeal ${ }^{\circ}$ Micro in the granulation and epithelialization of chronic ulcers of the lower limbs. Methods. Descriptive observational case series of 19 patients with diagnosis of chronic vascular ulcers of the lower limbs, treated at Medical Center Nuestra IPS and Medical Center Juan Pablo II in Bogotá between March 2018 and December 2018, who received PolyHeal ${ }^{\circledR}$ Micro as topical treatment for their lesions. Patients were assessed taking into account age, sex, ulcer size, pain, wound exudate, as well as granulation and epithelialization response. Results. In this series, the mean age of patients was 67.9 years, they were mostly females (84\%), and the most common location of ulcers was the internal malleolus (50\%). In terms of wound severity, $47 \%$ were found to be severe, $43 \%$ moderate, and $10 \%$ mild. The median time of lesion onset was 28 weeks, with a maximum of 2080 and a minimum of 8 weeks. In total, $87 \%$ of the ulcers were of venous etiology. After 12 weeks of treatment with Polyheal, ulcers showed a significative improvement of Wollina score means: $0.80 \pm 0.90-5.90 \pm 1.47 ;(p<0.000)$. Wound area mean at the start of treatment was $31.6 \mathrm{~cm}^{2}$, and at the end of treatment was $17.85 \mathrm{~cm}^{2}$, which is equivalent to a wound area surface reduction of $54.2 \%$, with a statistically significant $p$ value. The interquartile range showed a reduction of $64.3 \%$ in the central means of wounds. Seventy percent of the patients reached a granulation percentage greater than $70 \%, 17 \%$ of the lesions exhibited an improvement of $30-70 \%$, and there was an overall granulation improvement in $87 \%$ of the patients. Concerning epithelialization, $40 \%$ of patients reached a percentage higher than $70 \%$, and $17 \%$ of patients between $30 \%$ and $70 \%$. Treatment time was 12 weeks in $68 \%$ of cases, with an average response time of 8.1 weeks. Based on the visual analogue scale (VAS), a reduction in patients' perception of pain was achieved, dropping from an average of 6 (moderate to severe pain) to 2 (little pain), demonstrating an improvement in this regard. Fifty percent of the ulcers showed decreased exudate, resulting in a dry state. The patient satisfaction rate at the end of treatment was $89 \%$. Conclusion. The

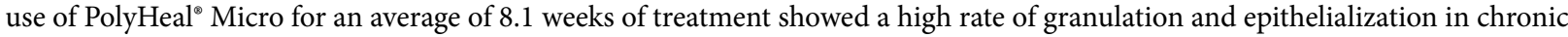
ulcers of the lower limbs, improving pain perception in these patients and generating a high degree of treatment satisfaction.

\section{Introduction}

The skin is considered the largest organ in most vertebrates, having a complex structure made up of three layers: the epidermis, the dermis, and the hypodermis, which under normal conditions have the ability to regenerate themselves [1]. However, its integrity may be affected by multiple causes, including chronic wounds, which may be extensive and deep, altering the biological healing process and modifying the complex series of interactions existing between the different types of cells, mediating molecules, and components of the extracellular matrix that allow the growth and adequate regeneration of tissues [2].

Chronic ulcers affect $1 \%$ of the adult population and $3.6 \%$ of people over 65 , rising to more than $5 \%$ in people over 80 [3]. 
These ulcers are due to different diseases; the most common ones are: chronic venous insufficiency constituting around $70 \%$ of leg ulcer presentations; chronic peripheral artery disease corresponding to 20\%; and diabetes mellitus which accounts for $85 \%$ of foot ulcers leading to peripheral neuropathy, often accompanied with arterial disease [4].

In the course of a lifetime, almost $10 \%$ of the population will develop a chronic wound, with a wound-related mortality rate of $2.5 \%$ [5], and although wound healing is a natural process of regeneration, the speed of healing in most cases of a chronic wound is slow, as $20 \%$ will not heal in less than 2 years [6], and approximately $8 \%$ will not heal after 5 years, reaching a health cost of 7 billion dollars in the USA annually [7]. It is therefore a significant cause of morbidity worldwide, causing prolonged hospitalizations, diminishing quality of life, resulting in incapacity to work, and representing great economic and social costs.

Professionals such as general practitioners, dermatologists, vascular surgeons, geriatricians, nurses, and sometimes psychiatrists deal with this issue on a daily basis [8].

Although currently there are a variety of films, topical medications, dressings and gels that help to close these wounds, technological advances have focused on understanding the biomolecular process of the wound and creating appropriate products for mediating the correct stage of the repair process that is disrupted, thereby generating excellent conditions for the skin to resume the process of healing and self-regeneration.

Based on the above, PolyHeal ${ }^{\bullet} \mathrm{Micro}$ has been developed, a substance composed of a suspension of negatively charged polystyrene microspheres (CPM) at a concentration of $4.5 \times 10^{6}$ microspheres $/ \mathrm{mL}$, suspended in glycerol and phosphate buffer in water for topical use. This makes it a quick and easy-to-apply product, which may be readily adapted to any area of the ulcer and whose mechanism of action generates cell adhesion, thus promoting processes of granulation and epithelialization in chronic wounds, and achieving in a short period, according to studies, a rapid response in the evolution of wounds [9]. In this way, after closure, comprehensive management of the patient's underlying pathology may be continued.

\section{Objective}

To describe the outcome and effectiveness of the use of PolyHeal $^{\circledR}$ Micro in the granulation and epithelialization of chronic ulcers of the lower limbs.

\section{Materials and Methods}

Descriptive observational case series.

3.1. Selection of Study Population. Patients were selected for the study based on the following criteria:

(1) Patients with chronic vascular ulcers (over 4 weeks) of the lower limbs of venous, arterial, mixed, or diabetic etiology.
TABLE 1: Ulcer classification based on diameter.

Ulcer classification based on diameter: [10]

(i) Mild: $<2 \mathrm{~cm}$

(ii) Moderate: $2-6 \mathrm{~cm}$

(iii) Severe: $>6 \mathrm{~cm}$

(2) All patients with the above criteria who were admitted for vascular surgery at Medical Center Nuestra IPS and at Medical Center Juan Pablo II in Bogotá between March 2018 and December 2018, and who attended the consultation.

Exclusion criteria:

(1) Patients with less than 4 weeks of evolution from onset of ulceration.

(2) Patients with chronic ulcers of the lower limbs with signs of infection.

To assess lesion severity, the longest side of the wound was taken as a parameter and classified according to Table 1.

3.2. PolyHeal ${ }^{\circledast}$ Micro Application Protocol. The number of bottles of PolyHeal $^{\oplus}$ Micro $7.5 \mathrm{ml}$ to be applied for each patient was determined based on the size of the ulcer, as defined by the outpatient specialist.

All patients received oral and written information about the treatment to be performed, and an informed consent was signed. The age, sex, anatomical location of the ulcers (internal malleolus, external malleolus, anterior tibia, and dorsum of the foot), time of lesion evolution, TIME model (tissue, infection/inflammation, moisture, and edge), wound pain according to the visual analogue scale, and size in terms of length and width $(\mathrm{cm})$ were recorded. These measures were taken at the start of, during, and after treatment.

All patients underwent the same application process, which was explained by the treating specialist both to the patient and to the caregiver in case there was one. They received in writing the step-by-step instructions for the healing of the wound, and follow-up was performed by the specialist every 8 days, where pictures were taken following this application protocol:

Specialist protocol (at the beginning of treatment and every 8 days)

(a) Clean the wound with $500 \mathrm{cc}$ of normal saline.

(b) Dry the wound with sterile gauze.

(c) Measure the lesion: length, width and depth in cephalocaudal direction.

(d) Evaluate lesion tissues according to TIME (viable tissue, signs of infection and inflammation, exudate, and perilesional edges).

(e) Take a photographic record.

(f) Shake the PolyHeal ${ }^{\oplus}$ Micro bottle.

(g) Apply PolyHeal ${ }^{\circledR}$ Micro to the entire lesion. 
(h) Cover lesion with gauze impregnated with PolyHeal ${ }^{\circ}$ Micro.

(i) No other type of product is applied around the lesion or its edges.

(j) Use dry gauze as a second dressing.

(k) Position cotton bandages and elastic bandage are positioned and compression is applied based on the presence and verification of pedal pulses, capillary filling and distal perfusion, fix them with adhesives.

(l) Give recommendations to patient and family member or caregiver on home care, and strengthen education in each session on nutrition, hygiene, administration of medications prescribed by the physician, physical activity, rest, leisure time, sexuality, bandage care at home, and warning signs.

(m) Check comfort of the bandage and general condition of the patient before release.

(n) Explain to the patient that he or she should clean the wound once a day every day of the week and that the bottle should be used in its entirety and discarded once the entire product has been applied.

(o) Schedule appointment within 8 days.

Patient and/or caregiver protocol for daily treatment

(a) Clean the wound with $500 \mathrm{cc}$ of normal saline.

(b) Dry the wound with sterile gauze.

(c) Shake the PolyHeal ${ }^{\circledR}$ Micro bottle.

(d) Apply PolyHeal ${ }^{\circ}$ Micro to the entire lesion.

(e) Cover lesion with gauze impregnated with PolyHeal ${ }^{\circ}$ Micro.

(f) No other type of product is applied around the lesion or its edges.

(g) Use dry gauze as a second dressing.

(h) Position cotton bandages and elastic bandage are positioned and compression is applied based on the presence and verification of pedal pulses, capillary filling and distal perfusion, fix them with adhesives.

(i) Discard the empty bottle of PolyHeal ${ }^{\circ}$ Micro.

(j) This process is carried out once a day, every day of the week.

The frequency of application was established at once a day, up to the total use of the formulated bottles.

\section{Statistical Analysis}

4.1. Demographic Characterization. The study population consisted of 19 patients with 30 vascular lesions in the lower limbs.

This population exhibited the following demographic characteristics:

The predominant sex of the study population is female, with more than 80\%, as shown in Figure 1 and Table 2.

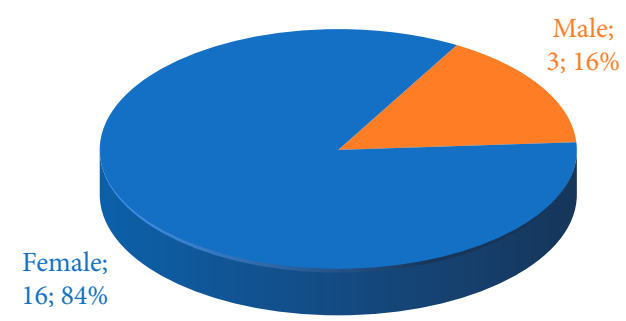

Figure 1: Population distribution by sex.

TABLE 2: Distribution by sex.

\begin{tabular}{lcc}
\hline Sex & No. & $\%$ \\
\hline Female & 16 & 84 \\
Male & 3 & 16 \\
Total & 19 & 100 \\
\hline
\end{tabular}

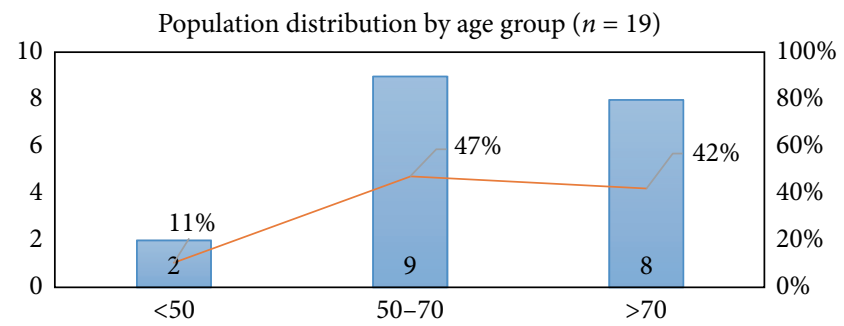

FIgURE 2: Population distribution by age group.

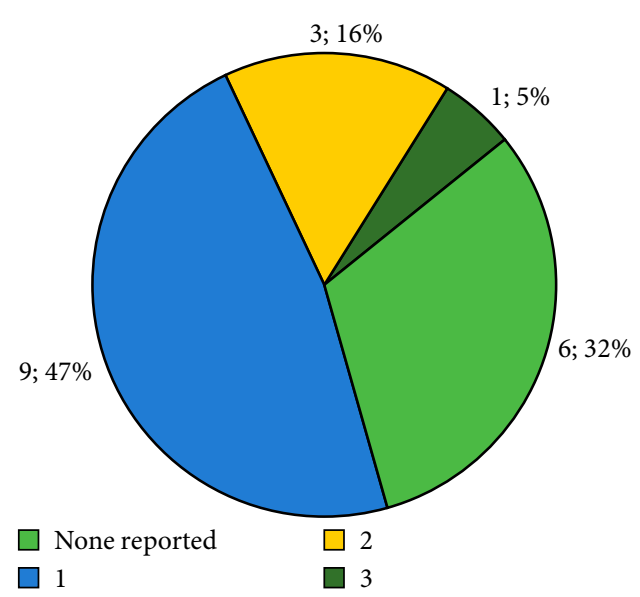

FIgURE 3: Number of associated pathologies per patient.

The average age in the study population was 67.9 years, with a maximum age of 90 and a minimum of 32 . The female group included the oldest individuals, with an average age of 68.5 years, compared to 63.7 years for men.

As may be observed in the following graph, the group of individuals over 50 years of age accounts for $89 \%$ of the population included in the study, and the group of 50-70 years constitutes the largest concentration of individuals. Figure 2.

Based on population characteristics, chronic non-communicable pathologies were found in $68 \%$ of the population, 
TABLE 3: Associated pathologies.

\begin{tabular}{ll}
\hline Associated pathologies & \\
\hline Vascular brain disease & 1 \\
Vascular brain disease + Hyperthyroidism & 1 \\
High blood pressure & 8 \\
High blood pressure + Heart disease & 1 \\
High blood pressure + Diabetes mellitus & 1 \\
High blood pressure + Diabetes mellitus + Obesity & 1 \\
None reported & 6 \\
\hline Total & 19 \\
\hline
\end{tabular}

TABle 4: Distribution by type of lesion.

\begin{tabular}{lcc}
\hline Type of lesion & No. & $\%$ \\
\hline Mixed ulcer & 4 & 13 \\
Venous ulcer & 26 & 87 \\
\hline Total & 30 & 100 \\
\hline
\end{tabular}

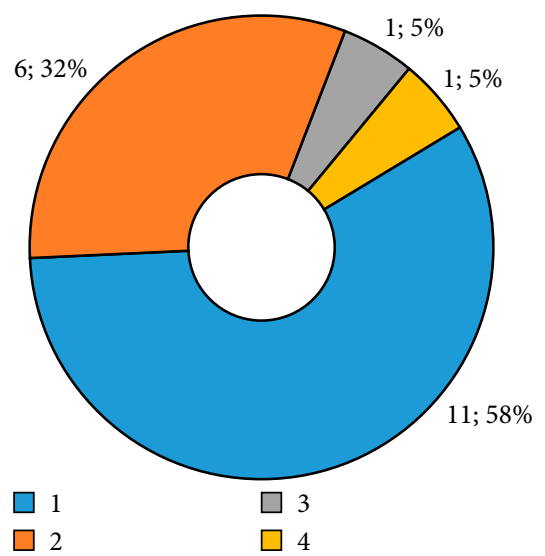

Figure 4: Number of lesions per patient.

with arterial hypertension being the most prevalent (Figure 3 and Table 3).

4.2. Lesion Characterization. Of the 30 lesions found in the 19 patients studied, the largest proportion corresponded to venous ulcers (Table 4).

In terms of the number of lesions per patient, it was found that more than $80 \%$ of the population had one or two lesions and $10 \%$ between 3 and 4 lesions (Figure 4 ).

4.3. Anatomical Location of Lesions. The ulcers were usually located anatomically on the lower and internal region of the lower limbs. In this study, these were mostly observed at the level of the internal and external malleoli with 70\%, followed by the anterior tibia with $19 \%$ (Table 5 ).

Regarding the time of onset of the vascular pathology in the study population, it was observed that $37 \%$ of the lesions were over 90 weeks old. The group of 8-40 weeks had the greatest number of lesions, accounting for $50 \%$ of the cases. In relation to lesion size, the largest lesions were concentrated in those with for more than 90 weeks, accounting for $26 \%$ of the cases (Table 6 and Figure 5).
TABLE 5: Anatomical location of lesions.

\begin{tabular}{lcc}
\hline & No. lesions & $\%$ \\
\hline Internal malleolus & 15 & 50 \\
External malleolus & 6 & 20 \\
Anterior tibia & 4 & 13 \\
Inner tibia and internal malleolus & 2 & 6 \\
Inner tibia & 1 & 3 \\
Inner tibia and external malleolus & 1 & 3 \\
Dorsum of the foot & 1 & 3 \\
\hline Total & 30 & 100 \\
\hline
\end{tabular}

TABLE 6: Time of evolution of lesions.

\begin{tabular}{lcccc}
\hline $\begin{array}{l}\text { Time of evolution } \\
\text { of lesions in weeks }\end{array}$ & Mild & $\begin{array}{c}\text { Lesion size } \\
\text { Moderate }\end{array}$ & Severe & Total \\
\hline $8-12$ & 3 & 4 & 1 & 8 \\
$12-40$ & & 4 & 3 & 7 \\
$40-90$ & & 2 & 2 & 4 \\
$>90$ & 3 & 13 & 8 & 11 \\
\hline Total & & & & 30 \\
\hline
\end{tabular}

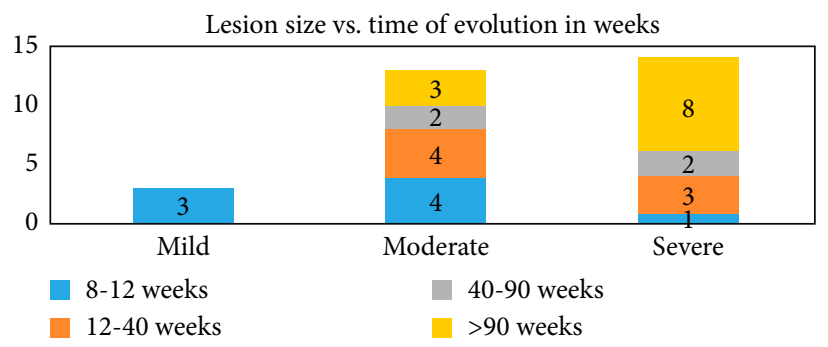

FIGURE 5: Time of evolution of lesions.

Table 7: Treatment time with PolyHeal $^{\circledR}$ Micro.

\begin{tabular}{lcc}
\hline $\begin{array}{l}\text { Treatment time } \\
\text { No. weeks }\end{array}$ & No. patients & $\%$ \\
\hline 3 & 1 & 5.0 \\
7 & 1 & 5.0 \\
9 & 3 & 16 \\
10 & 1 & 5.0 \\
12 & 13 & 68.0 \\
\hline Total & 19 & 100 \\
\hline
\end{tabular}

\section{Intervention Results}

Sixty-eight percent of treated patients received 12 weeks of treatment with PolyHeal $^{\circledR}$ Micro, whereas $16 \%$ received treatment for 9 weeks. The variation between the weeks of treatment was determined by the percentage of lesion granulation, since upon reaching expected granulation, the patient stopped receiving the product; the average time of appearance of results was 8.1 weeks (Table 7 ). 
TABLE 8: Wollina score.

\begin{tabular}{lccccccccc}
\hline & \multicolumn{2}{c}{ Week 0 } & \multicolumn{2}{c}{ Week 4 } & \multicolumn{2}{c}{ Week 8 } & \multicolumn{2}{c}{ Week 12 } \\
& Mean & SD & Mean & SD & Mean & SD & Mean & SD & $p^{*}$ value \\
\hline Granulation tissue (maximum 4) & 0.3 & 0.47 & 1.93 & 0.94 & 2.67 & 0.96 & 3.27 & 0.87 & 0.000000 \\
Color (maximum 2) & 0.6 & 0.62 & 1.23 & 0.50 & 1.43 & 0.57 & 1.77 & 0.43 & 0.000000 \\
Consistency (maximum 1) & 0 & 0 & 0.1 & 0.31 & 0.6 & 0.50 & 0.87 & 0.35 & 0.000000 \\
Total score wollina (maximum 7) & 0.87 & 0.90 & 3.13 & 1.46 & 4.67 & 1.63 & 5.90 & 1.47 & 0.000000 \\
Surface area $\left(\mathrm{cm}^{2}\right)$ & 31.36 & 31.82 & 25.58 & 28.77 & 20.52 & 27.25 & 17.85 & 26.56 & 0.000164 \\
\hline
\end{tabular}

${ }^{*}$ Statistically significant $(p<0.05)$.

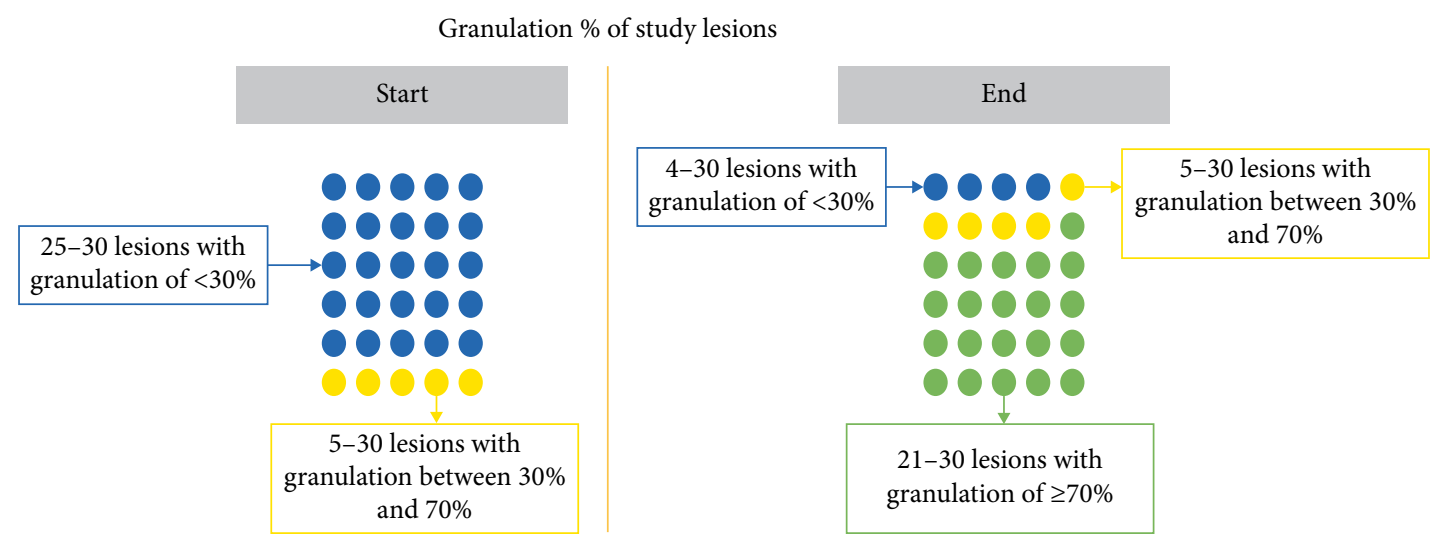

FIgURE 6: Results obtained with the use of PolyHeal ${ }^{\circledR}$ Micro: granulation.

\subsection{Weekly Assessment of the Wollina Score and Wound Area} Surface. After 12 weeks of treatment with Polyheal (Table 8), ulcers showed a significative improvement of Wollina score means: $0.80 \pm 0.90-5.90 \pm 1.47 ; \quad(p<0.000)$. Wound area mean at the start of treatment was $31.6 \mathrm{~cm}^{2}$, and at the end of treatment was $17.85 \mathrm{~cm}^{2}$, which is equivalent to a wound area surface reduction of $54.2 \%$, with a statistically significant $p$ value. The interquartile range showed a reduction of $64.3 \%$ in the central means of wounds.

A Friedman's test was used to compare non-parametric variables and a Student's $t$-test was used to compare parametric variables.

Friedman's test $X_{2 \mathrm{r}} 185.2$

$H_{0}$ hypothesis: Treatment does not improve ulcers' granulation.

$H_{1}$ hypothesis: Treatment improves ulcers' granulation.

$P=0.001$.

The alternative hypothesis is accepted: Polyheal improves ulcers' granulation.

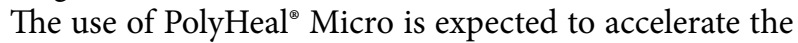
production of granulation tissue and lesion epithelization through negatively charged polystyrene microspheres, which have an effect on the inflammatory phase and improve the proliferative phase of healing for tissue repair.

In the study population, following the use of PolyHeal $^{\circledR}$ Micro, $70 \%$ of lesions reached a granulation level greater than $70 \%$ (Figures 6 and 7) and 17\% of wounds achieved a granulation percentage between $30 \%$ and $70 \%$; only $13 \%$ remained with a granulation percentage below $30 \%$.

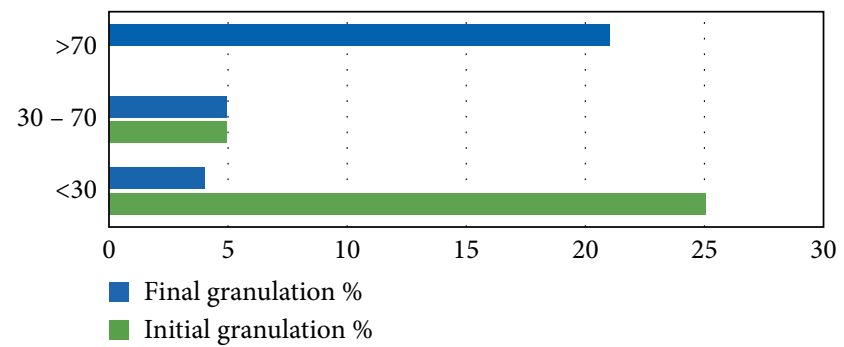

FIgURE 7: Comparison granulation \% at start and end of treatment.

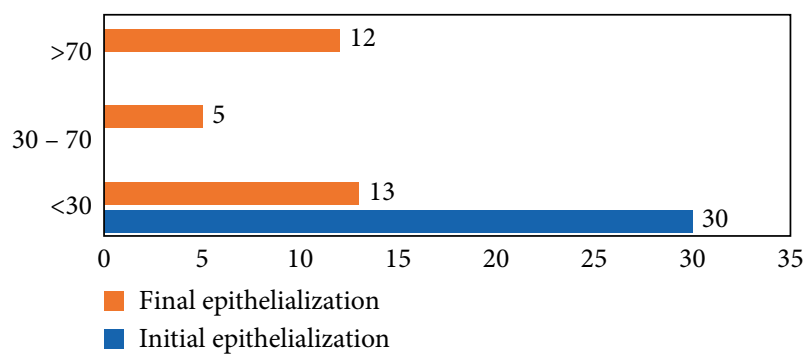

FIGURE 8: Comparison epithelialization \% at start and end of treatment.

As for the epithelialization of lesions at the end of treatment with PolyHeal $^{\circledR}$ Micro, $57 \%$ of the lesions in the study reached an epithelialization level between $30 \%$ and $70 \%$ (Figure 8). 


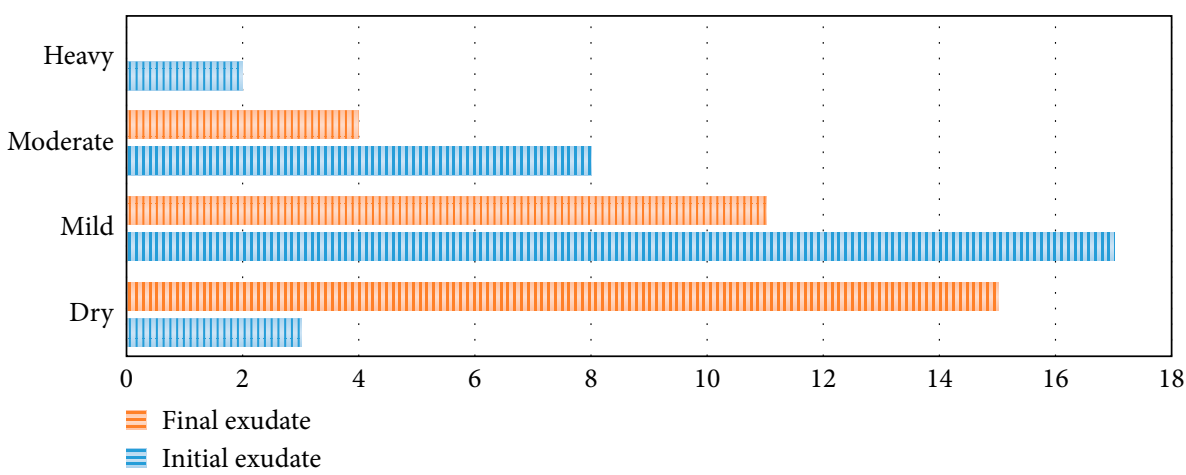

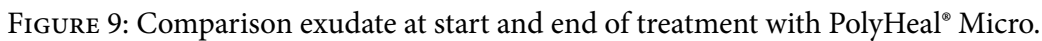

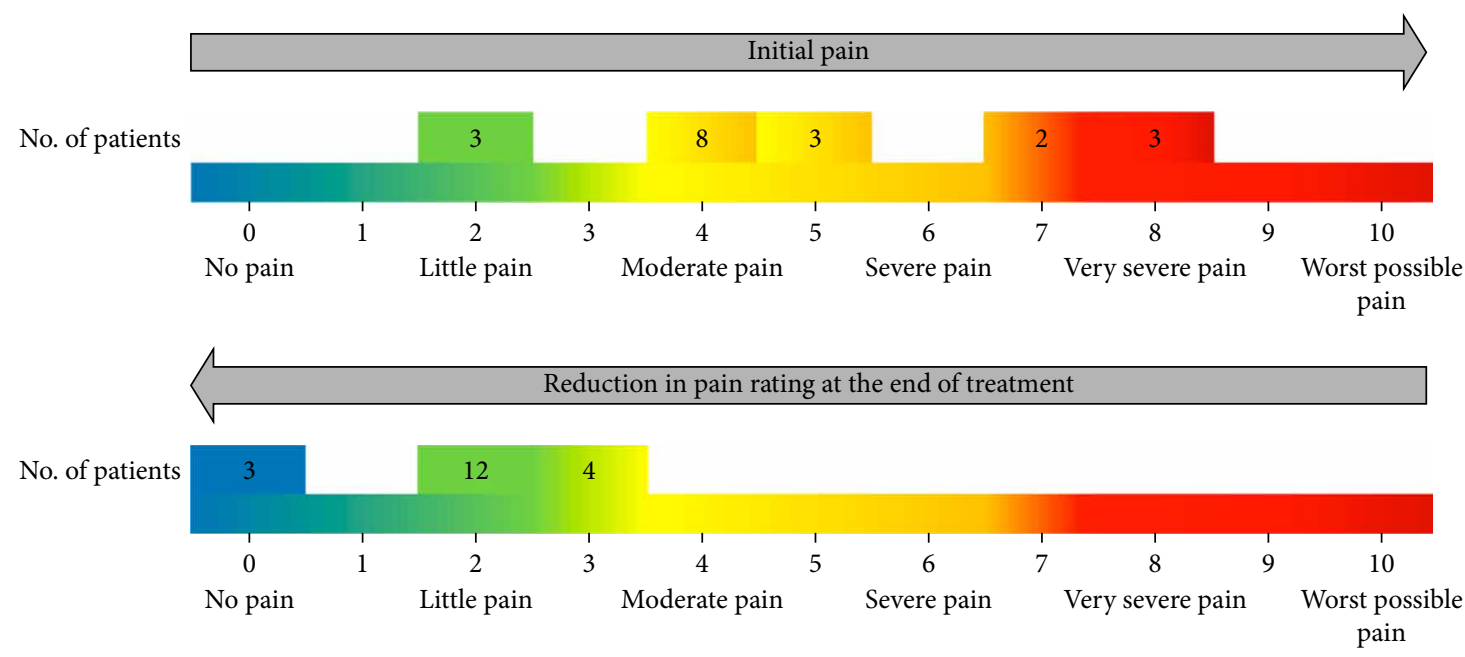

FIGURE 10: Comparison initial pain vs. final pain rating.

TABLE 9: Final results by lesion size.

\begin{tabular}{lcccccc}
\hline $\begin{array}{l}\text { Lesion } \\
\text { size at } \\
\text { start of } \\
\text { treatment }\end{array}$ & \multicolumn{3}{c}{ Final granulation } & \multicolumn{3}{c}{ Final epithelialization } \\
\hline $\begin{array}{l}\text { Mild } \\
(<2 \mathrm{~cm})\end{array}$ & 1 & $30-70$ & $>70$ & $<30$ & $30-70$ & $>70$ \\
$\begin{array}{l}\text { Moderate } \\
(2-6 \mathrm{~cm})\end{array}$ & 2 & 2 & 9 & 5 & 2 & 6 \\
$\begin{array}{l}\text { Severe } \\
(>6 \mathrm{~cm})\end{array}$ & 1 & 3 & 10 & 7 & 3 & 4 \\
Total & 4 & 5 & 21 & 13 & 5 & 12 \\
\hline
\end{tabular}

When evaluating the final granulation and epithelialization results, the following was found (Table 9):

(i) Mild lesions reached $67 \%$ of granulation and epithelialization greater than $70 \%$.

(ii) Moderate lesions reached $69 \%$ of granulation, and $46 \%$ reached an epithelialization greater than $70 \%$.

(iii) Severe lesions reached $71 \%$ of granulation, and $29 \%$ reached an epithelialization greater than $70 \%$.

\section{Statistical Testing}

6.1. For Lesion Epithelialization. Wilcoxon's statistical test was carried out with a $95 \%$ confidence interval, noting that due to the sample size of the statistic $T$ where the data are adjusted to a normal distribution, the statistic $Z$ is calculated to accept the hypotheses presented.

$\mathrm{H}_{0}$ : No significant changes in wound size before and after the use of Polyheal

$H_{\mathrm{A}}$ : Significant changes in wound size before and after the use of Polyheal

$$
\begin{aligned}
& H_{0}=M_{\text {ed }}=0, \\
& H_{\mathrm{A}}=M_{\text {ed }} \neq 0,
\end{aligned}
$$

$$
Z=\frac{t-N(N+1) / 4}{\sqrt{N(N+1)(2 N+1) / 24}}=N(0.1) \quad Z=-4.45 .
$$

For the analysis of the study sample, the alternative hypothesis was accepted, which indicates that the positive results obtained in terms of wound size with the use of PolyHeal ${ }^{\circledR}$ Micro are statistically significant with $\alpha=0.05$. 
TABLe 10: Patient satisfaction results.

Patient satisfaction with treatment from study admission

Increase

No variation

Concerning the lesion exudate, a significant improvement was noted since at the end of sn no heavy exudate was observed, and $50 \%$ of the wounds became dry (Figure 9).

During the study, the visual analogue scale was applied to patients on admission and at the end of treatment. On admission, patients rated the level of lesion pain, reaching an average of 6 or severe pain. Once the intervention was concluded, the measurement was performed again, revealing a reduction in pain in all patients who, on average, reached a score of two. This corresponds to little pain, which proves to be a significant change in this regard (Figure 10).

\section{Statistical Testing}

7.1. To Measure Pain. Wilcoxon's statistical test was carried out with a $95 \%$ confidence interval, noting that due to the sample size of the statistic $T$ where the data are adjusted to a normal distribution, the statistic $Z$ is calculated to accept the hypotheses presented.

$\mathrm{H}_{0}$ : No significant changes in wound pain severity before and after the use of PolyHeal ${ }^{\circledR}$ Micro.

$H_{\mathrm{A}}$ : Significant changes in wound pain severity before and after the use of PolyHeal $^{\circledR}$ Micro.

$$
\begin{gathered}
H_{0}=M_{\text {ed }}=0, \\
H_{\mathrm{A}}=M_{\text {ed }} \neq 0 . \\
Z=\frac{t-N(N+1) / 4}{\sqrt{N(N+1)(2 N+1) / 24}}=N(0,1) \quad Z=-4.54 .
\end{gathered}
$$

For the analysis of the study sample, the alternative hypothesis was accepted, which indicates that the results obtained in terms of pain reduction with the use of PolyHeal $^{\circledR}$ Micro are statistically significant with $\alpha=0.05$.

According to the results obtained, $89 \%$ of the intervened patients showed an increase in the level of satisfaction, compared to previous treatments and to study admission (Table 10).

\section{Discussion}

Wound healing involves a complex physiological process aimed at restoring the integrity of the skin, as any impairment in its barrier function generates disturbance in the homeostasis and general well-being of the patient [11].

Chronic wounds continue to be a great medical challenge and, despite the technological advances and various treatments for their cure, they are still pathologies that generate great disability, morbidity and mortality, directly impacting health expenditure. They are a major public health problem because their treatment is expensive and may involve prolonged periods of hospitalization and additional surgical procedures.

Thus, chronic wounds constitute one of the greatest health care problems affecting the health system. They decrease the quality of life of those who develop them and their caregivers [12] and represent a high economic cost, based on wound treatment period, which according to Fernández [13], may vary between 150 and 180 days to achieve an effective recovery, coupled with the time spent by the nursing professionals responsible for the various treatments.

Based on the literature, if a wound does not respond to standard care (a response typically defined as 30\% reduction in wound size for venous leg ulcers and 50\% reduction for diabetic foot ulcers, within 4 weeks after treatment initiation), a change in treatment and use of specialized technology [14, 15 ] is indicated. Additionally, it should be borne in mind that the treatment of chronic ulcers has a double aspect: The underlying disease and local wound treatment [16], as the risk of such lesions recurring must always be minimized.

Finally, the constant technological development of different useful products for wound healing offered by the market, together with the progressive development of knowledge about molecular biology of wound healing, has demonstrated the importance of approaching this pathology with a treatment that not only reduces healing time and improves the patient's quality of life and morbidity, but also reduces costs. As such, PolyHeal ${ }^{\oplus}$ Micro is a substance that has an ability to replicate the functions of the extracellular matrix and promotes cell growth and proliferation, achieving a biochemical balance in the lesion. It increases the synthesis of collagen and the number of keratinocytes and endothelial cells, promoting the wound granulation process. It may be considered as a useful product in reducing healing time, with optimal results in improving the wound healing process.

\section{Conclusion}

The use of PolyHeal ${ }^{\circledR}$ Micro promotes the formation of granulation tissue, by increasing the percentage of wound healing. This therapy constitutes a major advance in the care of chronic wounds and has the potential to significantly improve the condition of most patients, without causing adverse events and showing good tolerability. The therapy is adjuvant to the management of the underlying pathology of the patient. It is an innovative and easy-to-use treatment that has demonstrated favorable results in terms of reduction of healing time, pain, and size of chronic wounds of the lower limbs, achieving a granulation percentage of more than $70 \%$ in $70 \%$ of patients, with a percentage of overall improvement in granulation of $87 \%$.

Concerning epithelialization, $40 \%$ of patients reached a percentage higher than $70 \%$, and $17 \%$ of patients between $30 \%$ and $70 \%$. The overall percentage of epithelialization was $57 \%$. Additionally, a significant decrease in pain was achieved in most patients, as well as a reduction in the production of exudate, thus obtaining a treatment satisfaction index of $89 \%$. 


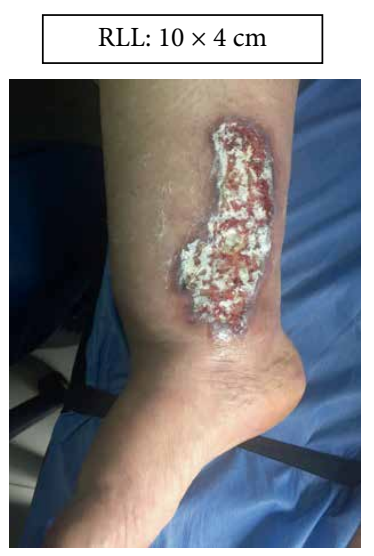

FIgURE 11: Start. Lower right limb.

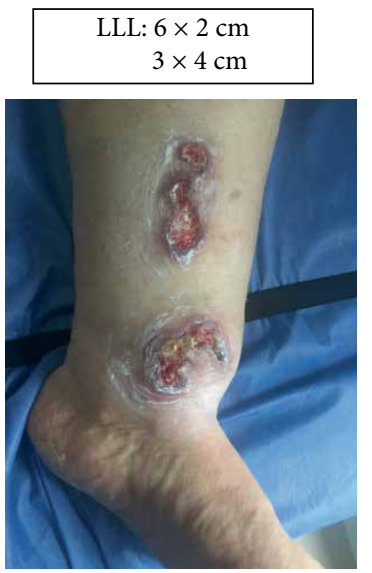

FIGURE 12: Start. Lower left limb.

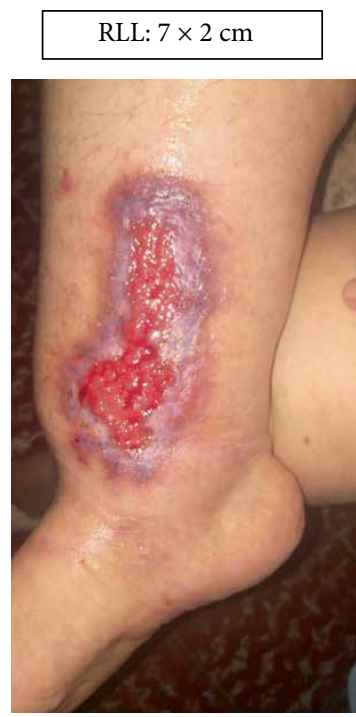

FIGURE 13: Week 4. Lower right limb.

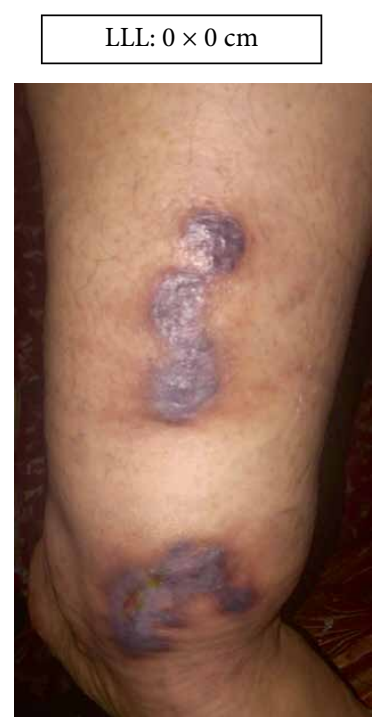

FIGURE 14: Week 4 . Lower left limb.

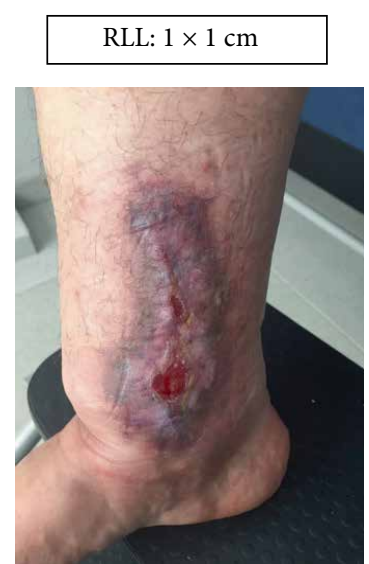

FIGURE 15: Week 9. Lower right limb.

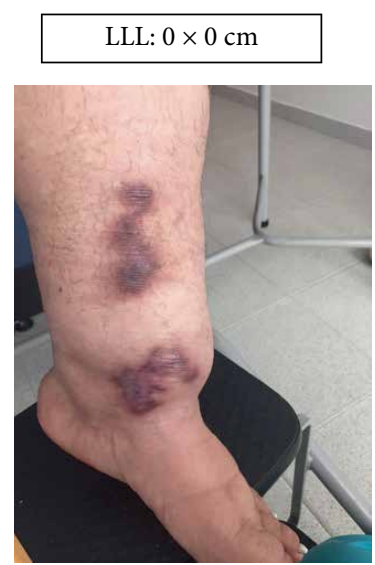

FIGURE 16: Week 9. Lower left limb. 


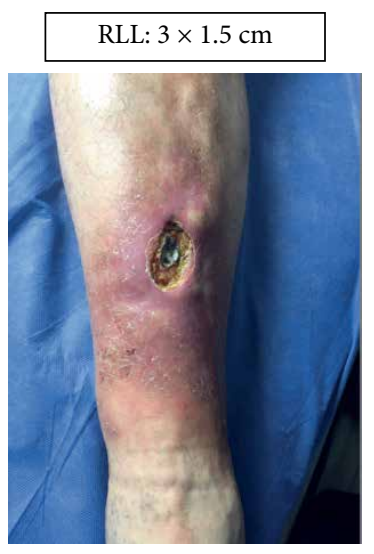

FIGURE 17: Start. Lower right limb.

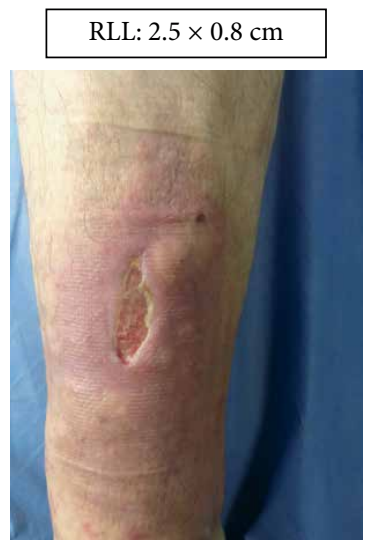

FIGURE 18: Week 5. Lower right limb.

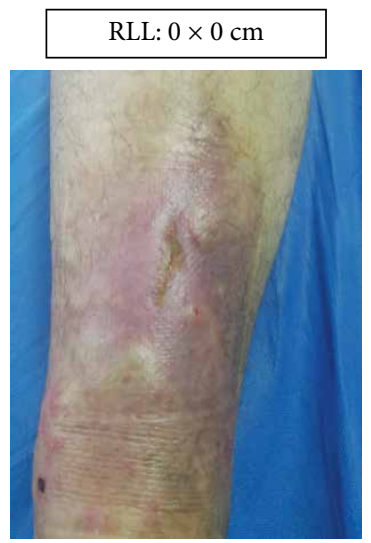

Figure 19: Week 10. Lower right limb.

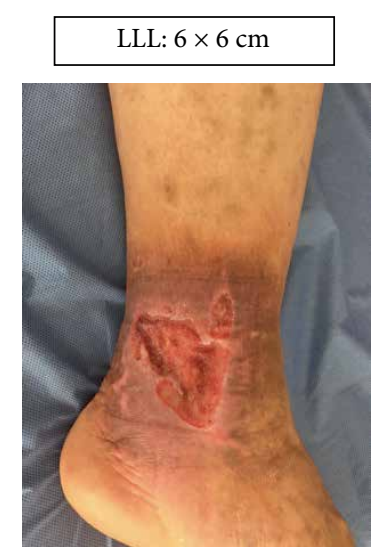

FIGURE 20: Start. Lower left limb.

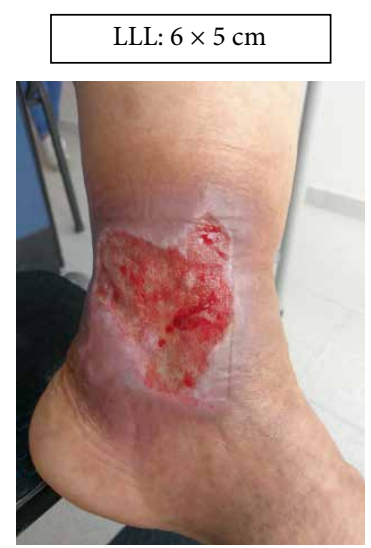

FIgURE 21: Week 6. Lower left limb.

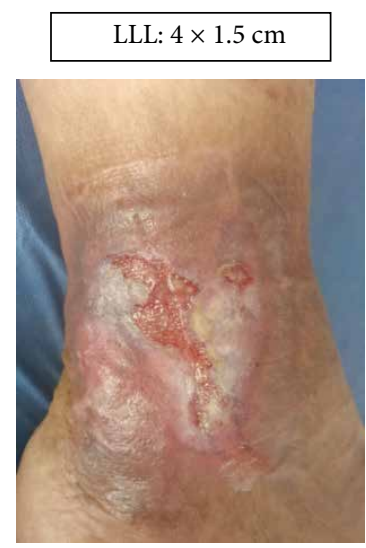

FIGURE 22: Week 12. Lower left limb. 


\section{Clinical Cases}

RLL: Lower right limb.

LLL: Lower left limb.

Case 1.

Start (Figures 11 and 12).

Week 4 (Figures 13 and 14).

Week 9 (Figures 15 and 16).

Case 2 (Figures 17-19).

Case 3 (Figures 20-22).

\section{Data Availability}

The data used to support the findings of this study are available from the corresponding author upon request.

\section{Conflicts of Interest}

The authors declare that they have no conflicts of interest.

\section{References}

[1] L. E. Valencia-Gómez, "Natural polymers aposites for skin regeneration," Mexican Journal of Biomedical Engineering, vol. 37, no. 3, 2016.

[2] A. E. Rivera and J. M. Spencer, "Clinical aspects of full-thickness wound healing," Clinics in Dermatology, vol. 25, no. 1, pp. 39-48, 2007.

[3] R. Rayner, K. Carville, J. Keaton, J. Prentice, and X. N. Santamaria, "Leg ulcers: atypical presentations and associated co-morbidities," Wound Practice and Research, vol. 17, no. 4, pp. 168-185, 2009.

[4] S. V. Agale, "Chronic leg ulcers: epidemiology, aetiopathogenesis, and management," Ulcers, vol. 2013, pp. 1-9, 2013.

[5] C. S. Sasanka, "Venous ulcers of the lower limb: where do we stand?" Indian Journal of Plastic Surgery, vol. 45, no. 2, pp. 266-274, 2012.

[6] J. Vuerstaek and T. Vainas, "State-of-the-art treatment of chronic leg ulcers," Journal of Vascular Surgery, vol. 44, no. 5, 2006.

[7] M. Velasco, "Diagnostic and therapeutic aspects of ulcers," Practical Dermatology Valencia Spain, vol. 102, no. 10, pp. 749-840, 2011.

[8] M. Escobar, J. Henao, M. Wolff, S. Estrada, and L. Marina, "Chronic lower limb ulcers treatment with autologous skin equivalent and larvae debriding," Latreia, vol. 20, no. 4, 2007, Medellín.

[9] J. Govrin, K. Leonid, E. Luger, J. Tamir, and G. l. Zeilig, R. Shafir, "New method for treating hard-to-heal wounds: clinical experience with charged polystyrene microspheres," Wounds UK, vol. 6, no. 4, 2010.

[10] T. F. O’Donnell Jr., M. A. Passman, W. A. Marston, W. J. Ennis, M. Dalsing, and R. L. Kistner, "Management of venous leg ulcers: clinical practice guidelines of the society for vascular surgery ${ }^{\oplus}$ and the American venous forum," Journal of Vascular Surgery, vol. 60, no. 2, 2014.

[11] C. Guarín-Corredor, P. Quiroga-Santamaría, and N. S. Landínez-Parra, "Wound healing process of skin, endogenous fields related and chronic wounds," Journal of the Faculty of Medicine, vol. 61, no. 4, pp. 441-448, 2013.
[12] L. Hernández, M. P. Hernández, and R. M. Suárez, "Chronic wounds treated in an emergency service of primary health care," Nursing Global, vol. 13, no. 35, 2014, Murcia.

[13] J. I. Fernández-Montequín, "Wounds of difficult healing," Cuban Journal of Angiology and Vascular Surgery, vol. 13, no. 1, pp. 1-10, 2012.

[14] S. Zimny and M. Fohl, "Healing times and prediction of wound healing in neurophatic diabetic foot ulcers. A prospective study," Experimental and Clinical Endocrinology \& Diabetes, vol. 113, no. 2, pp. 90-93, 2005.

[15] D. J. Margolis, L. Allen Taylor, O. Hoffstadt, and J. A. Berlin, "The accuracy of venous leg ulcer prognostic models in a wound care system," Wound Repair and Regeneration, vol. 12, no. 2, pp. 163-168, 2004.

[16] J. C. Moreno-Gimenez, M. Galán-Gutierrez, and R. JiménezPuya, "Treatment of chronic ulcers," Dermosifiliographic Records, vol. 96, no. 3, pp. 133-202, 2005. 


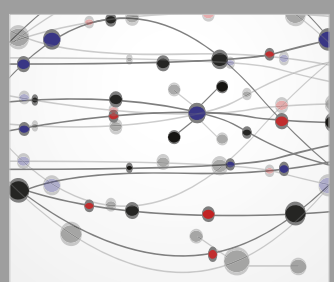

The Scientific World Journal
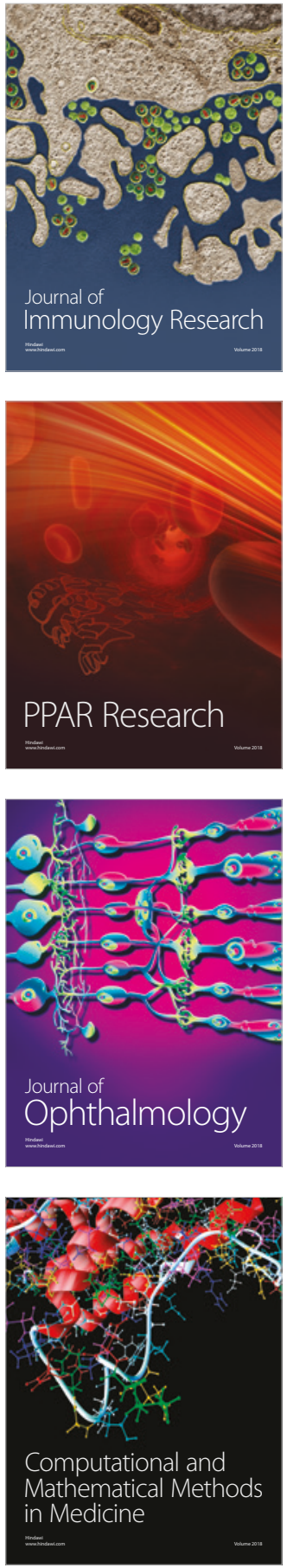

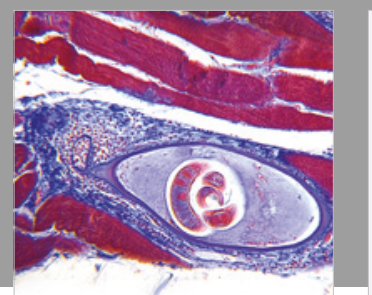

Gastroenterology Research and Practice

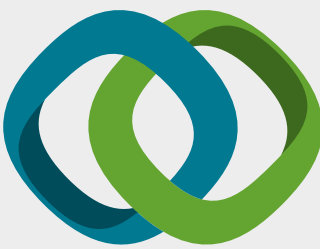

\section{Hindawi}

Submit your manuscripts at

www.hindawi.com
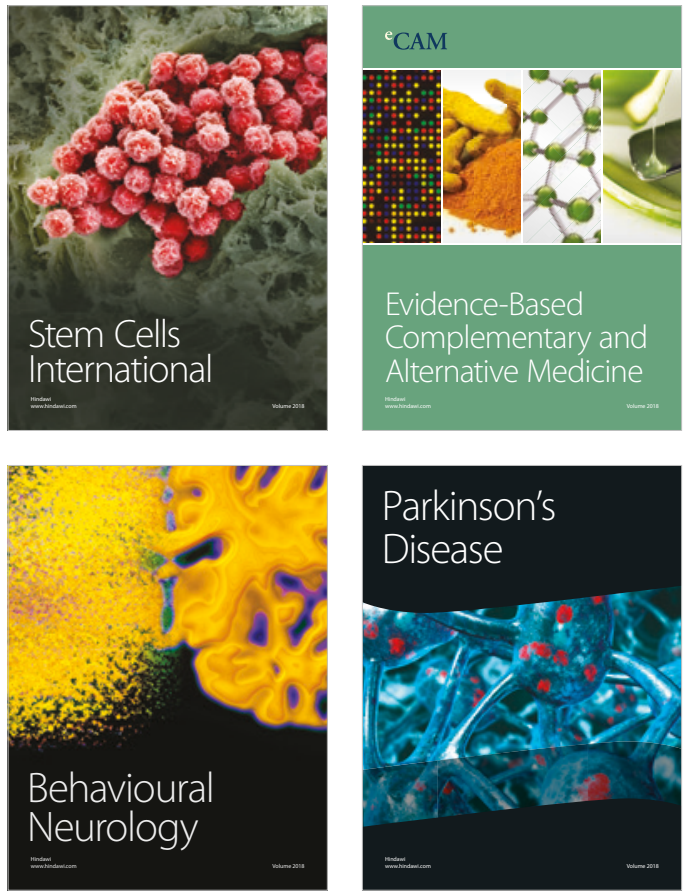

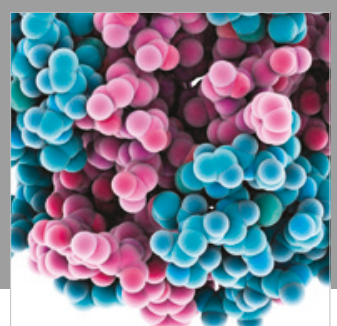

ournal of

Diabetes Research

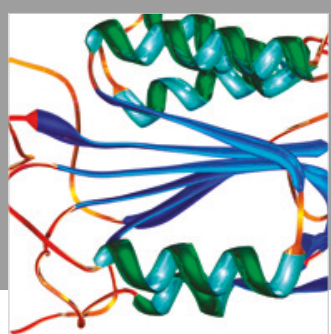

Disease Markers
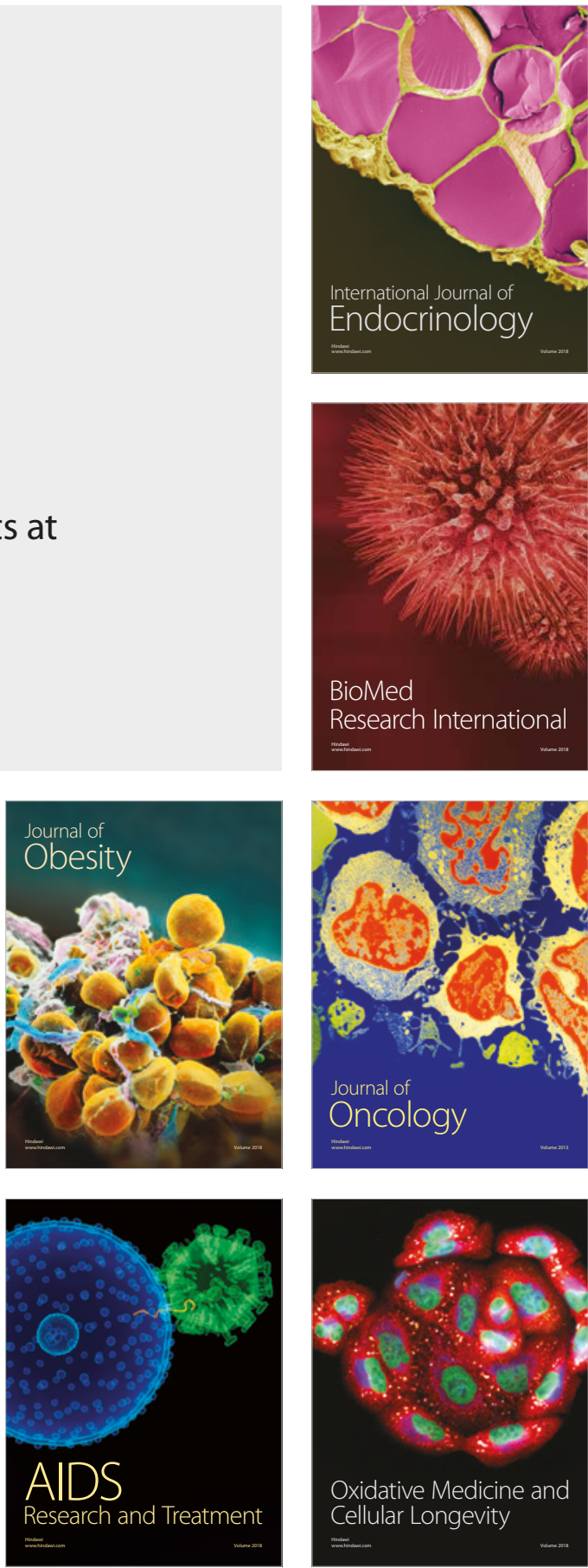